\title{
Gastric ulcer caused by immunoglobulin G4-related diseases: A case report
}

\author{
Xiaowen Li, Songbai Zheng, Li Xiao, Daming Shen, Danian Ji \\ Department of Gastroenterology, Huadong hospital, Fudan University, Shanghai, China \\ Correspondence: Songbai Zheng. Address: Department of Gastroenterology, Huadong hospital, Fudan University, \\ Shanghai, China. Email: fdhuadong@163.com \\ Received: February 25, 2015 \\ Accepted: June 18, 2015 \\ Online Published: June 28, 2015 \\ DOI : $10.5430 /$ crim.v2n3p35 \\ URL: http://dx.doi.org/10.5430/crim.v2n3p35
}

\section{Abstract}

Objective: To enhance the diagnosis and treatment of Immunoglobulin G4-related gastric disease.

Method: One case of IgG4-related gastric disease in our hospital was presented and analyzed.

Result: Patients with IgG4-related gastric disease usually showed nonspecific clinical symptoms such as abdominal discomfort, dull pain, anorexia, nausea and vomiting, anemia, weight loss, etc. By gastroscopy, we could divide IgG4-related gastro intestinal disease into two categories: organ enlargement and ulceration, whose histopathological features consisting of abundant infiltration of IgG4 positive lymphocytes, plasma cells, formation of lymph follicle. The lesion could spreading to every layer of the stomach, nerve, minute vessels and regional lymph nodes, which only existed in stomach that were not associated with other IgG4-related diseases. The diagnosis of the disease may refer to Japanese IgG4-related disease diagnostic criteria. We should identify it to exclude gastric cancer clinically. Treatment with steroids was given priority to, and surgery could be a complementary method.

Conclusion: The incidence of IgG4 related-gastric disease is rare, and it is not difficult to diagnose and treat by analysis of the limited cases. It is of utmost importance for us to raise vigilance, consider IgG4-related disease and especially to accumulate more cases, explore its pathogenesis, clinical characteristics if possible.

\section{Keywords}

IgG4-related disease, Gastric, Plasmocyte, Malignancy, Ulcer

\section{I ntroduction}

Immunoglobulin G4-related disease (IgG4RD) is a recently described entity, which is characterised by organ enlargement or nodular lesions consisting of abundant infiltration of lymphocytes and variable degrees of fibrosis. Immunohistochemistry stains showed a dense lymphoplasmacytic infiltrate rich in IgG4 ${ }^{+}$plasma cells. IgG4RD was first documented in an autoimmune pancreatitis (AIP) IgG4-positive plasma cells may involve not only the pancreas, but also in almost every organ system, such as the bile duct, gallbladder, salivary gland, thyroid gland, lungs, stomach, colon, liver, retroperitoneum, kidney, prostate and lymph nodes ${ }^{[1]}$. Nevertheless, IgG4-related mass lesions in the stomach have been rarely reported. We encountered a 63-year-old man with large gastric ulcer of suspicious malignancy, while it was characterized pathologically as an IgG4-related autoimmune fibrosclerosing mass. 


\section{Case report}

A 63-year-old man hospitalized with a two-year history of left epigastric discomfort, whose symptoms aggravated in 3 months with poor appetite and body weight loss. He had deteriorated discomfort after drinking, and alleviated when take ranitidine orally. The patient denied abdominal pain, diarrhea, fevers, night sweats, hematemesis or melena. His past health history included hypertension and alcoholic hepatitis. There was no history of rheumatic disease. He had no allergies, and was not taking any medication. He had a history of tobacco smoking and alcohol consuming socially more than 30 years, whereas no family history of cancer or autoimmune disease. On medical examination, he appeared normal, with a temperature of $36.5{ }^{\circ} \mathrm{C}$, blood pressure $115 / 75 \mathrm{mmHg}$, and pulse 78 beats/min. The patient $\mathrm{s}$ abdomen was soft, without tenderness or distension. 13C-acetate breath test initiated a positive result for Helicobacter pylori infection, but he didn't receive any eradication therapy. The patient underwent an electrogastroscopy, and two large ulcer lesions were noted, which were suspicious for gastric cancer (Borrmann III), one $40 \mathrm{~mm} \times 35 \mathrm{~mm}$ in the fundus (see Figure 1), another $30 \mathrm{~mm} \times 30 \mathrm{~mm}$ in gastric angle, with white tongue coating. Gastric mucosa which surrounding ulcers showed a mild-to-moderate atrophic change with intestinal metaplasia.

Figure 1. Endoscopic finding: an approximately $40 \mathrm{~mm}$, large ulcer lesions covered with white tongue coat in the fundus, which was suspicious for gastric cancer (BorrmannIII)

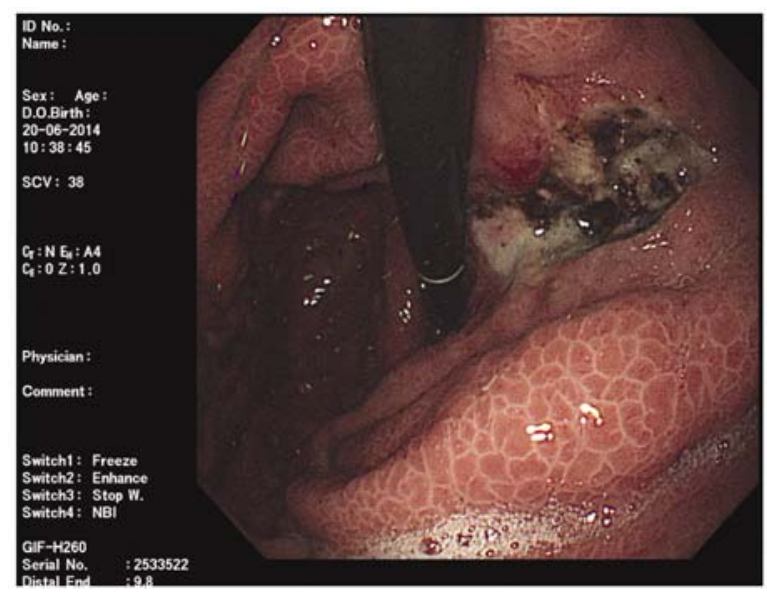

Multiple sets of biopsies were sampled from the ulcer edge during this hospitalization, which showed evidence of chronic ulceration with markedly acute inflammation, with lymphocytic infiltrating among the lamina propria and mild hyperplastic mucosa of partial gland; there was no evidence of malignancy. Abdominal computerised tomography (CT) scan revealed thickened stomach wall of lesser gastric curvature, with multiple perigastric lymph nodes measuring from $3 \mathrm{~mm}$ to $17 \mathrm{~mm}$, which were considered as malignant (see Figure 2). The CT scan detected no abnormity in the liver, gall bladder, pancreas together with spleen.

Figure 2. Computed tomography scan: thickened stomach wall of lesser gastric curvature, with multiple perigastric lymph nodes measuring from $3 \mathrm{~mm}$ to $17 \mathrm{~mm}$, which were considered as malignant

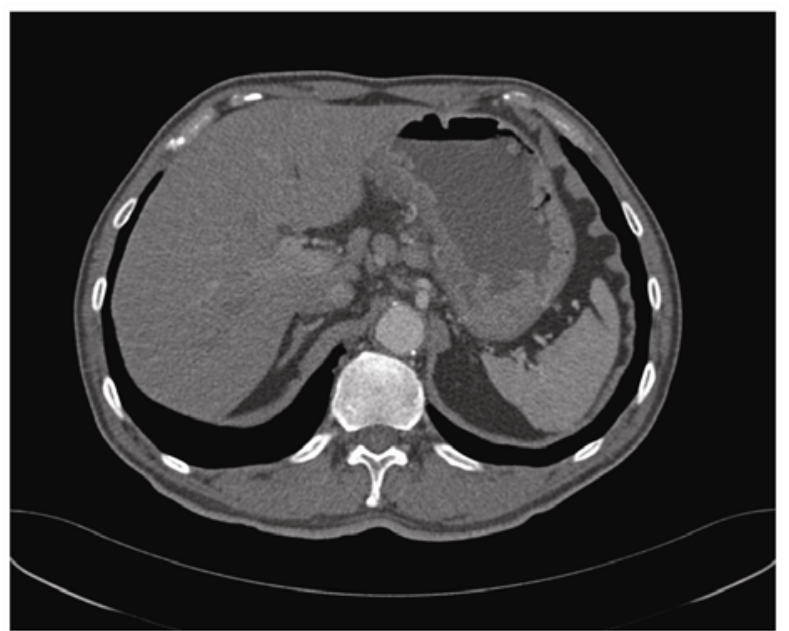

ISSN 2332-7243 E-ISSN 2332-7251 
To avoid the progress and identify its malignancy, the patient subsequently went on to have exploratory laparoscopic resection with total gastrectomy, after which he made a full recovery and was discharged on day 14 postoperatively.

Macroscopic examination of the resection specimen showed a $40 \mathrm{~mm} \times 35 \mathrm{~mm}$ mucosal ulcer with markedly thickened stomach wall of $15 \mathrm{~mm}$. Gastric mucosa around the ulcer was abnormal granular and oedematous. Histopathological examination of the specimen revealed abundant dense lymphoplasmacytic infiltrating and submucosal fibrosis (see Figure 3). Multiple lymphoid follicles showing prominent germinal centers are observed occasionally. Occasional obliterated veins and nerves were densely infiltrated by lymphoplasmacytes, with atrophic smooth muscle and hyperplasia of fibrous tissue. Immunostaining presented diffusely distributed immunoglobulin G4 (IgG4)-positive cells within the lesion with an increased numbers of IgG4 ${ }^{+}$plasma cells 50-60 cells per high power field (HPF). The amount of IgG4 ${ }^{+}$plasma cells was over $50 \%$ of total $\mathrm{IgG}^{+}$plasma cells (see Figure 4), which provided appropriate histological diagnosis of IgG4RD. Immunohistochemical stains were negative for CK7, CK, Cam5.2, CK20, ALK, CD30, SMA, S100, while positive for PGM1.

Corticosteroid therapy and the management of side effects were considered, but the patient, who was asymptomatic, declined this option. After 5 months` recovery (December 2014), he remains well.
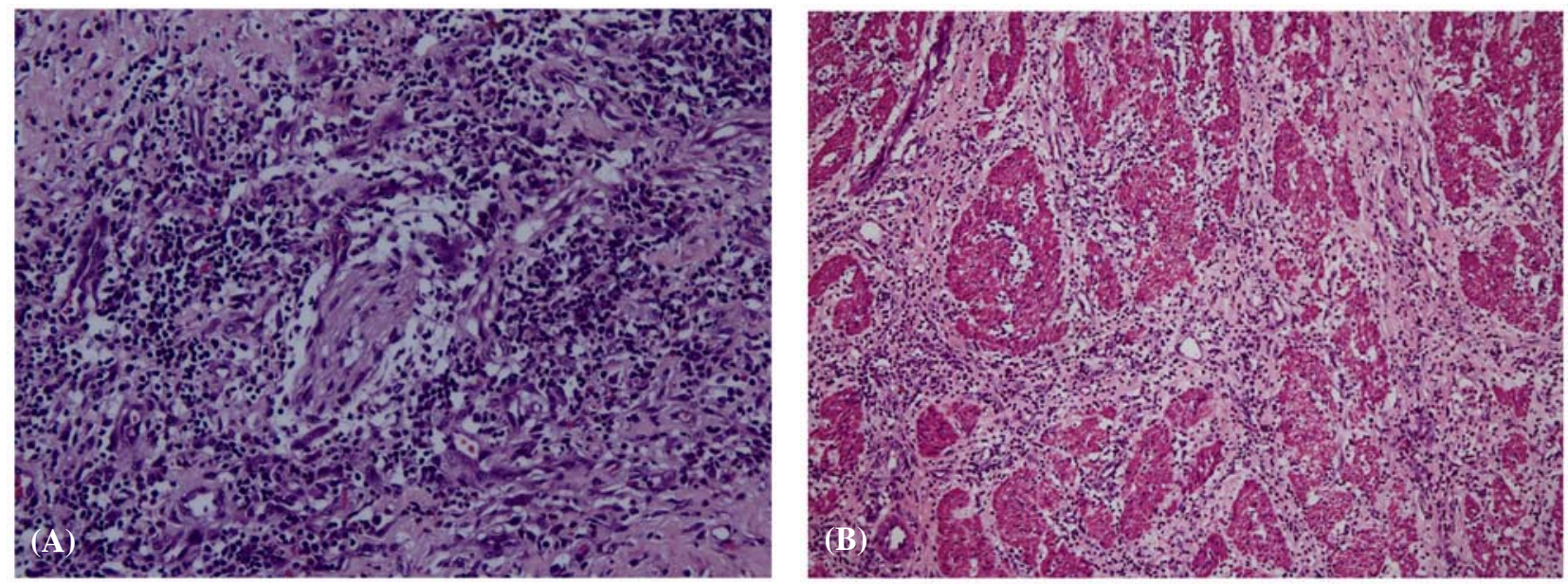

Figure 3. (A) Several nerve fibers within the ulcer base are completely infiltrated by lymphoplasmacytic cells (H\&E; orig. mag. $\times 200$ ). (B) Within the ulcer base are abundant fibrous stroma and numerous lymphoplasm cells (H\&E; orig. mag. $\times 200$ ). (C) Several veins and arteries within the ulcer base are completely obliterated by aggregated inflammatory cell infiltration (but without current luminal obliteration) (H\&E; orig. mag. $\times 200$ )

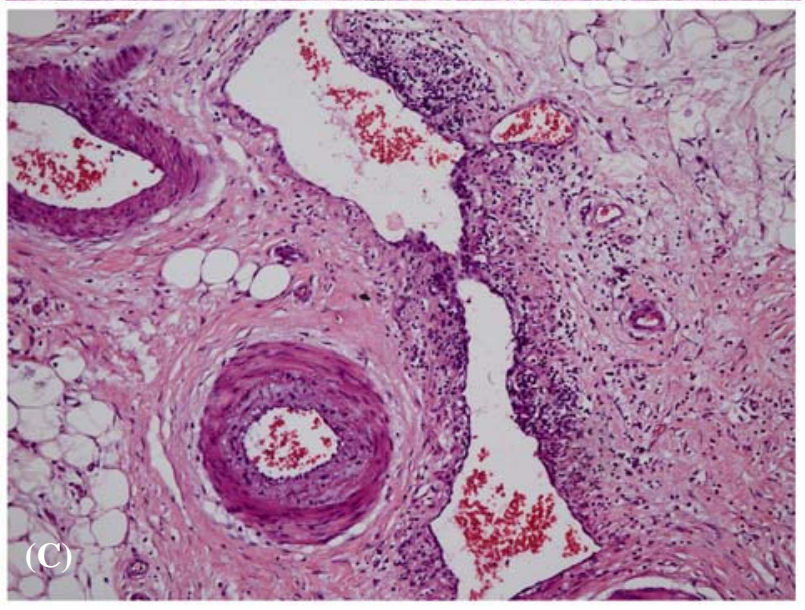



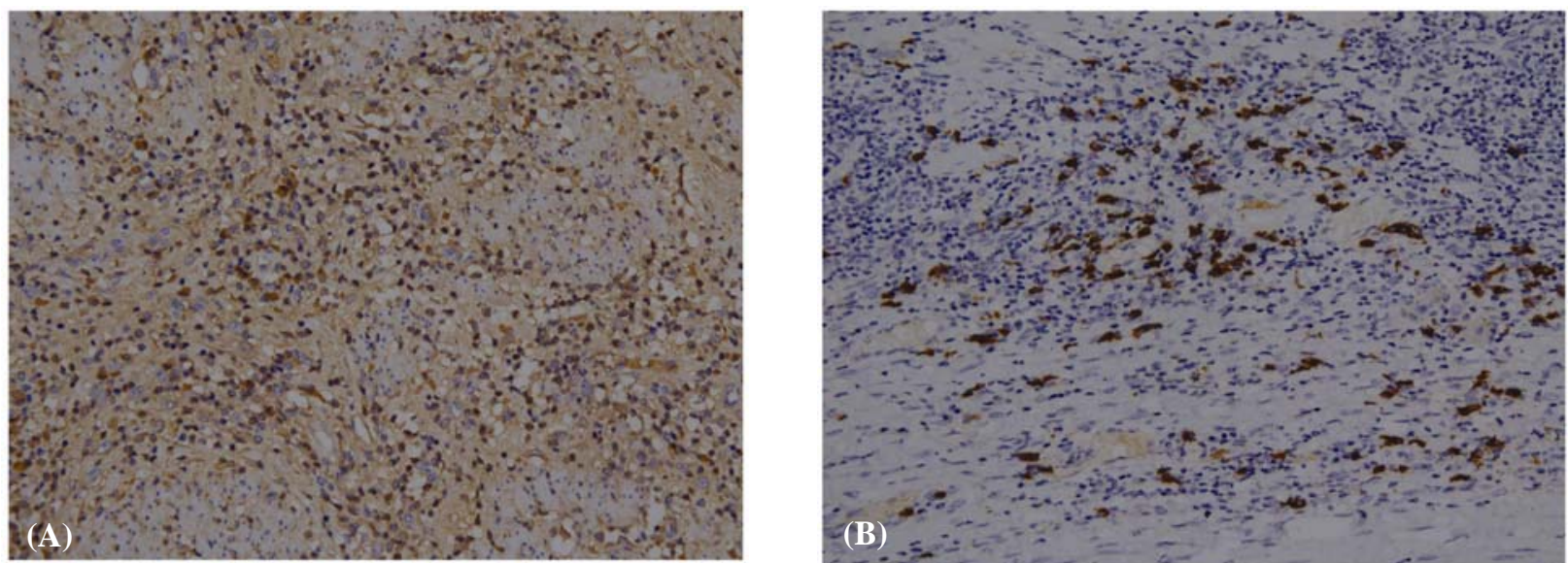

Figure 4. (A) IgG immunohistochemistry demonstrated IgG positive plasma cells (orig. mag. ×200). (B) IgG4 immunohistochemistry demonstrated IgG4 positive plasma cells (orig. mag. $\times 200$ )

\section{Discussion}

IgG4-related autoimmune refractory ulcer is a newly recognized fibro-inflammatory condition that characterized by varied features: organ enlargement or nodular lesions consisting of abundant infiltration of lymphocytes; varying degrees of fibrosis; and usually dramatically but not always elevated serum IgG4 concentrations.

Recent literature found that IgG4-related disease in stomach showed nonspecific clinical symptoms such as nausea, vomiting, abdominal pain, anemia, weight loss, which was also different under gastrioscopy and CT. By reviewing past papers, we could divide IgG4-related gastrointestinal disease into two categories: one is gastrointestinal ulceration showing prominent thickening of the wall of the esophagus and stomach ${ }^{[2,3]}$, with abundant infiltration of IgG4-positive plasma cells spreading submucosal, the other is an IgG4-related pseudotumor showing polypoid or mass-like lesions that occurring in gastrointestinal regions such as the stomach ${ }^{[4,5]}$, colon, and major duodenal papilla. Bateman et al. ${ }^{[2]}$ described a case with gastric ulcer that is refractory to proton pump inhibitors (PPI); histological examination revealed marked submucosal fibrosis, with a partly storiform pattern, associated with the mucosal ulceration. The present case showed similar manifestations, while Rollins et al. ${ }^{[4]}$ reported another different case with a large polypoid lesion in the gastric body. We current consider these lesions to be IgG4-related gastric disease. However, further studies should be conducted to identify this disease.

Comprehensive clinical diagnostic criteria for IgG4-related disease were proposed by Japanese research committee in 2011, which including (1) diffuse/localized swelling or mass in a single or multiple organs; (2) elevation of serum IgG4 levels $\geqslant 1350 \mathrm{mg} / \mathrm{L}$; (3) histological findings: fibrosis, usually storiform in character and dense lymphoplasmacytic infiltration. The percentage of IgG4-positive plasmocytes was above 40\%, and IgG4 counts required for the diagnosis differ among affected organs, ranging from 10 to 200 cells/hpf. If the patient presented with single organ that wasn't satisfy comprehensive diagnosis standard, he/she can be diagnosed with organ specificity. Most solitary IgG4-related gastric lesions exist in stomach that are not associated with other IgG4-related diseases appear to be difficult to diagnose. This case highlights how such a lesion can masquerade as a type of dysplastic and malignant tumor endoscopically and radiographically. Although its incidence is rare, it is of utmost importance for us to rule out malignancy, consider IgG4-related disease when mucosa gastric ulceration is identified and to obtain a definitive preoperative diagnosis if possible. Hence, several unneeded invasive therapy, including resection can be avoided.

It is our case that first report of a patient who showing gastric ulcer in Chinese. The histopathological features were classical for IgG4-related disease and are the same as those described within affected tissues from other anatomical sites. 
The histopathological entity of IgG4-autoimmune gastric ulcer is becoming recognized more frequently in a variety of locations, requiring treatment with steroids rather than surgery.

\section{References}

[1] Stone JH, Zen Y, Deshpande V. IgG4-related disease. The New England journal of medicine. 2012; 366(6): 539-551. PMid:22316447 http://dx.doi.org/10.1056/NEJMra1104650

[2] Bateman AC, Sommerlad M, Underwood TJ. Chronic gastric ulceration: a novel manifestation of IgG4-related disease? Journal of clinical pathology. 2012; 65(6): 569-570. PMid:22259178 http://dx.doi.org/10.1136/jclinpath-2011-200565

[3] Fujita T, Ando T, Sakakibara M, et al. Refractory gastric ulcer with abundant IgG4-positive plasma cell infiltration: a case report. World journal of gastroenterology: WJG. 2010; 16(17): 2183-2186. PMid:20440861 http://dx.doi.org/10.3748/wjg.v16.i17.2183

[4] Rollins KE, Mehta SP, O'Donovan M, et al. Gastric IgG4-Related Autoimmune Fibrosclerosing Pseudotumour: A Novel Location. ISRN gastroenterology. 2011; 2011: 873087. PMid:21991533 http://dx.doi.org/10.5402/2011/873087

[5] Kim do H, Kim J, Park do H, et al. Immunoglobulin G4-related inflammatory pseudotumor of the stomach. Gastrointestinal endoscopy. 2012; 76(2): 451-452. PMid:21981816 http://dx.doi.org/10.1016/j.gie.2011.07.061

[6] [Comprehensive diagnostic criteria for IgG4-related disease (IgG4-RD), 2011. Research Program of Intractable Disease provided by the Ministry of Health, Labor, and Welfare of Japan]. Nihon Naika Gakkai zasshi The Journal of the Japanese Society of Internal Medicine. 2012; 101(3): 795-804. 